\title{
PROCESOS DE DETERMINACIÓN DE LA CAPACIDAD JURÍDICA, TRASTORNOS PSICÓTICOS Y COMPETENCIA PARA VOTAR
}

GUARDIANSHIP, PSYCHOTIC DISORDERS AND CAPACITY TO VOTE

Matías Juan Martínez ${ }^{1,2}$.

\section{Resumen:}

Resumen: El objetivo del presente artículo es ofrecer una aproximación al estado de la cuestión de la actuación en los procesos judiciales de determinación de la capacidad jurídica en personas afectadas por trastornos psicóticos y su competencia para votar. Para ello se ofrece centralmente una revisión de la temática con especial énfasis en los aportes desde la psiquiatría, vinculados entre otros aspectos a los modelos de evaluación de capacidad para toma de decisiones, y la comunicación de un breve estudio descriptivo de los resultados obtenidos en procedimientos realizados en un Juzgado de Familia de la Provincia de Buenos Aires en el período comprendido desde marzo de 2014 a diciembre de 2016.

Palabras clave: psiquiatría; psiquiatría forense; espectro de esquizofrenia y otros trastornos psicóticos; evaluación de la discapacidad; personas con discapacidad mental.

\begin{abstract}
:
The article is aimed to offer an approach of the state of the art of the practices in legal ability determination proceedings in people affected by psychotic disorders and their competency to vote. Therefore, we offer a revision of the subject with special emphasis in the contribution from psychiatry, linked with capacity evaluation models for the decision-making process, and the report of a brief descriptive study based on the results of legal proceedings carried on by a Family Court in Buenos Aires Province from March 2014 to December 2016.
\end{abstract}

Keywords: psychiatry; forensic psychiatry; schizophrenia spectrum and others psychotic disorders; mental disabled persons.

1 Médico. Especialista en Psiquiatría y Medicina Legal. Perito de Equipo Técnico Juzgado de Familia de la Provincia de Buenos Aires.
2 Email de contacto: matiasjuanmartinez@gmail.com 


\section{Introducción}

Los cambios promovidos en las prácticas evaluativas en los procesos de Determinación de la Capacidad Jurídica han elevado a un plano destacado la requisitoria desde el poder judicial de la opinión pericial acerca de la competencia para votar de las personas afectadas por trastornos mentales graves.

Esta cuestión puede movilizar profundos interrogantes de naturaleza ético-axiológica y epistemológicos en los profesionales, ya sean de un ámbito forense o asistencial, a los que puede serles formulado dicho requerimiento. Así es planteado con humildad por un prestigioso psiquiatra de nuestro medio: “¿quién soy yo psiquiatra, para opinar acerca de si un ciudadano puede votar o no?, porque yo podría informar sobre una funcionalidad psíquica, sobre un cierto grado de inteligencia o sobre un razonamiento lógico o bizarro. Pero utilizar el discurso científico de las psicodisciplinas para avanzar sobre tales cuestiones merece una prudente reflexión ${ }^{-(1)}$. Esta encrucijada resume vectores sociales, terapéuticos y normativos, que, aunque ya han realizado un cierto despliegue temporal, permanecen en tensión, y posee un carácter novedoso que no había sido objeto de interés en recientes manuales de psiquiatría forense nacionales 0 monográficos sobre insania e inhabilitación ${ }^{(2-5)}$ ni tratado en el "Instrumento de Relevamiento - Capacidad Jurídica" distribuido por la Dirección Nacional de Salud Mental y Adicciones en $2012^{(6)}$. Sí podemos señalar que se haya incluida dentro de la ambiciosa Clasificación Internacional del Funcionamiento, de la Discapacidad y de la Salud de la Organización Mundial de la Salud ${ }^{(7)}$ en su capítulo de Vida Comunitaria, Social y Cívica-Vida política y ciudadanía dentro de Actividades y Participación y en Servicios, Sistemas y Políticas de Gobierno dentro de Factores Ambientales.

\section{Antecedentes remotos}

Las obras clásicas de psiquiatría forense y medicina legal en nuestro país, que son frondosas en sus desarrollos y teorizaciones sobre la materia de las insanias, no explicitan inclinación a atender los pormenores de la exclusión del derecho al sufragio activo de este colectivo de personas ${ }^{(8-12)}$. Tampoco en forma comparada consta el abordaje de la cuestión de marras en obras señeras de España ${ }^{a(13)} 0$ Alemania $^{(14)}$. Respecto a Italia, De Sanctis y Ottolenghi advierten de la complejidad de la tarea de asesorar en procedimientos de incapacitación civil: "La capacidad civil bien lejos de 'no pescar muy a fondo en abismo misterioso de la pasión y de la ética' (Tanzi), interesa a todavía más aptitudes psicológicas que la capacidad criminal o imputabilidad. Quién no puede ver la complejidad de aptitudes psicológicas que son interesadas en las variadas y múltiples manifestaciones de la vida civil, del simple acto de autoridad paterna o marital a aquellos de disponer de los propios ingresos y después de muerte de los bienes propios, eso, sin adentrarse por el campo del derecho público que comprende las manifestaciones múltiples y variadísimas de la vida política y social?"(15) y más adelante prosigue afirmando "no se puede abstraer al considerar la capacidad civil del sentimiento ético individual y social. Esto que se impone en las manifestaciones resguardantes del tratamiento de los intereses individuales, se impone tanto más cuando la capacidad civil se desenvolverá en el ámbito de la familia y de la sociedad (derecho político)" (15). Y en Francia, Henri Claude únicamente reseña que "el ejercicio de los derechos civiles es independiente del ejercicio de los derechos políticos, los que se adquieren y conservan conforme a las leyes constitucional y electoral. Ahora bien; la pérdida del ejercicio de los derechos civiles, lo más frecuentemente obedece a la existencia de trastornos mentales, que colocan al individuo mayor en tales condiciones de inferioridad, que hay motivo para protegerle mediante una especial tutela" ${ }^{(16)}$.

La excepción la encontramos en Degenerados y Degeneración de Francisco De Veyga ${ }^{(17)}$. Aquel texto, que en una lectura anacrónica hiere nuestra sensibilidad de época y puede ser calificado de brulote, aborda directamente el punto y transcribiremos del mismo un fragmento extenso. Allí define inicialmente "Entendiendo por degenerado (...) a todo ser decaído o anómalo, incompleto o inadaptado" (217p.). Posteriormente delimita cinco grupos de degenerados: 1-"La escoria apagada (...) todo el conjunto de seniles, de enfermos crónicos, lisiados, dementes, mendigos, vagabundos, a quienes ya no alcanza ningún recurso preventivo ni ningún tratamiento correctivo, reconstructivo o educativo" (356p.), 2-"La escoria agresiva (...) delincuentes habituales o profesionales de orden diverso, sueltos o recluidos. lunfardos, tahúres, rameras, etc.-" (360p.), 3- "los viciosos: toxicópatas, jugadores, pervertidos sexuales" (364p.), 4- "menores abandonados o desviados" (375p.) y 5-"retardados, insuficientes e incultos" (380p.).Y dentro de las estrategias de actuación plantea "debiera decretarse la supresión de la capacidad civil y electoral definitiva para los primeros, transitoria o parcial para los segundos. Son incapaces, jurídicamente hablando, todos ellos y por tanto deben estar sometidos a la acción de la tutela. Una tutela vigilante a la vez que represiva. (...) La supresión de la capacidad civil es medida de urgencia, salvadora, para psicópatas, ya ensayada por otras legislaciones, en algunas de las cuales se llega hasta la anulación del matrimonio o al divorcio absoluto. La supresión de la capacidad electoral es, a su vez, una 
medida de higiene moral y de buen sentido que disminuirá, si no concluiría con el tráfico de votos, en nuestro país." (374p.)

\section{Evolución normativa y conceptual}

Ya desde la fundacional "ley Sáenz Peña” 8.871 de 1912 se estableció en su artículo 2 que "Están excluidos del padrón electoral: 1. Por razón de incapacidad: a) Los dementes declarados en juicio" y 2. "Por razón de su estado y condición: (...) d) Los dementes y mendigos, mientras estén recluidos en asilos públicos, y en general, todos los que se hallen asilados en hospicios públicos ó estén habitualmente a cargo de congregaciones de caridad.". Y según el antiguo Código Civil previamente vigente desde 1871 en su artículo 141 "Se declaran dementes los individuos de uno y otro sexo que se hallen en estado habitual de manía, demencia o imbecilidad, aunque tengan intervalos lúcidos, o la manía sea parcial." ${ }^{-b(18)}$.

En continuidad con aquel contexto explicaba Nerio Rojas ${ }^{(19)}$ que existen "tres fundamentos de la interdicción: económico, biológico y mixto" y opinaba que el "fundamento biológico de la interdicción es un criterio objetivo, sólo busca comprobar el estado morboso. Es un fundamento psiquiátrico que consiste en que el perito y el juez resuelvan el hecho concreto y probado en el juicio de si el sujeto es o no es un enfermo mental. Comprobar la existencia o inexistencia de la alienación mental es la base de ese criterio concreto. (...) es el criterio actual de nuestro artículo 141 "(44-46p) y conforme a ello ofrecía su definición "La alienación mental es un estado morboso de grupos heterogéneos. (...) Prácticamente, ¿Qué es un alienado? Si ustedes quieren que les dé una definición poco técnica pero verídica, diré que "alienado" es para nosotros el enfermo que tenemos derecho a internar en el hospicio; el que no tenemos derecho a internar por su estado mental, ese no es alienado. (26-27p.) (...) La alienación mental es un trastorno general y persistente de las funciones intelectuales, cuyo carácter patológico es ignorado o mal comprendido por el enfermo, y que impide la adaptación lógica y activa a las normas del medio ambiente, sin provecho para sí mismo ni a la sociedad." (28p.) A la par de este planteo taxativo, el citado autor deslizaba una crítica a la legislación vigente por no haber previsto los estados intermedios o fronterizos.

Aquella modalidad de aproximación a través del diagnóstico (que había establecido una suerte de férrea asociación con un esquema dicotómico de capaz-incapaz absoluto) ha dejado de ser la preferida y se favorece en la actualidad una aproximación clínica por funcionamiento que impone la evaluación detallista y dimensional en clave de habilidades funcionales y competencias ${ }^{c}$ específicas siendo estas tantas como las actividades a realizar y consideradas de interés relevante -y dentro de ellas comienza a atenderse a la competencia para votar- $(20,21)$.

En lo normativo se sucedieron numerosos cambios, vertiginosamente en años recientes, que impactaron en el paradigma vigente y las modalidades de trabajo actuales (incontables papers han reseñado dicho proceso y fatigado las novedades del abordaje interdisciplinario, de la reevaluación exigida en un plazo trianual, del modelo social de discapacidad y de la gradualidad de la capacidad de obrar; entre ellos en una selección seguramente arbitraria e insuficiente: Palacios ${ }^{(22)}$, Martínez y Daray ${ }^{(23)}$, y Toro Martínez ${ }^{(1)}$ ). Resulta imperioso resumir entonces los mojones sentados por el proyecto de reforma del Código Civil por Ramón Carrillo de 1950 que pretendía incorporar la figura de "seminterdicción que consistirá en la privación del ejercicio de algunos derechos por déficit de la capacidad, o en la restricción de dicho

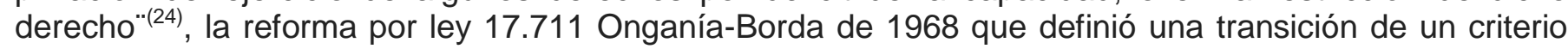
"de diagnóstico psiquiátrico puro" a uno "mixto psiquiátrico-jurídico" a través de aquello de "que por causa de enfermedades mentales no tengan aptitud para dirigir su persona o administrar sus bienes" e incorporó en su artículo 152bis la figura intermedia de la inhabilitación declarable "1- a quienes por embriaguez habitual o uso de estupefacientes estén expuestos a otorgar actos jurídicos perjudiciales a su persona o patrimonio; 2- a los disminuidos en sus facultades cuando sin llegar al supuesto previsto en el art 141 de este código, el juez estima que del ejercicio de su plena capacidad pueda resultar presumiblemente daño a su persona o patrimonio; 3- A quienes por la prodigalidad en los actos de administración y disposición de sus bienes expusiese a su familia a la pérdida del patrimonio", y de las más cercanas Convención sobre los Derechos de las Personas con Discapacidad (aprobada por la ONU en 2006, leyes nacionales 26.378 de 2008 y 27.044 de 2014) que forma parte de nuestro bloque de constitucionalidad, la ley nacional de Derecho a la Protección de la Salud Mental 26.657 de 2010, y la ley nacional 26.994 de 2014 de nuevo Código Civil y Comercial de la Sanción que entraría en vigencia en agosto 2015.

En el área electoral la modificación legal más trascendente para nuestro campo de estudio se produjo por la ley nacional 26.571 de 2009, que modificó el inciso a del artículo 3 del código electoral (ley 19.945 de 1972 y decreto 2135/83) limitando la exclusión del padrón exclusivamente a aquellos "dementes declarados tales en juicio" al suprimir la porción de su texto que también lo estipulaba para "aquellos que, aun cuando no lo hubieran sido, se encuentren recluidos en establecimientos públicos" d. Esta 
modificación fue destacada como positiva por el Comité sobre los Derechos de las Personas con Discapacidad de las Naciones en su informe de 2012 de observaciones sobre Argentina pero continuó manifestando su preocupación porque "las reformas al Código Electoral no hayan incluido a las personas declaradas incapaces por vía judicial, las cuales continúan siendo privadas del derecho al voto" (25). Y en el mismo sentido se pronunció en abril de 2016 en su dictamen el procurador fiscal ante la Corte Suprema de Justicia de la Nación en el expediente aún en curso "F,HO s/ artículo 152 ter Código Civil": "ya no es posible mantener la categoría jurídica de demente, en los términos del artículo 3, inciso a, del Código Electoral, puesto que el Código Civil y Comercial de la Nación, norma posterior específica de igual jerarquía, ha diseñado un sistema en el que no se admite la limitación de la capacidad de los sujetos sino únicamente de sus actos específicos" y para que su "exclusión del padrón electoral resultara válida, se debió concluir que carecía de capacidad para realizar ese acto político específico" (26).

Hacia el año 2000 los estudios identificaban solo 4 países, Canadá, Irlanda, Italia y Suecia, que no tenían establecidas restricciones para el voto de las personas con discapacidad mental. Una docena de años más tarde su número se ha amplificado a 16 e incluye dentro de Sudamérica a Bolivia y Ecuador ${ }^{\mathrm{e}}$ (27).

Entonces, actualmente, los textos que debemos conocer central e ineludiblemente de nuestro plexo normativo para nuestra materia de estudio son el art. 32 del CCyCN que dice "Persona con capacidad restringida y con incapacidad. El juez puede restringir la capacidad para determinados actos de una persona mayor de trece años que padece una adicción o una alteración mental permanente o prolongada, de suficiente gravedad, siempre que estime que del ejercicio de su plena capacidad puede resultar un daño a su persona o a sus bienes. En relación con dichos actos, el juez debe designar el o los apoyos necesarios que prevé el artículo 43, especificando las funciones con los ajustes razonables en función de las necesidades y circunstancias de la persona. El o los apoyos designados deben promover la autonomía y favorecer las decisiones que respondan a las preferencias de la persona protegida. Por excepción, cuando la persona se encuentre absolutamente imposibilitada de interaccionar con su entorno y expresar su voluntad por cualquier modo, medio o formato adecuado y el sistema de apoyos resulte ineficaz, el juez puede declarar la incapacidad y designar un curador" y art. 29 de la CDPD que consagra "Participación en la vida política y pública Los Estados Partes garantizarán a las personas con discapacidad los derechos políticos y la posibilidad de gozar de ellos en igualdad de condiciones con las demás y se comprometerán a: Asegurar que las personas con discapacidad puedan participar plena y efectivamente en la vida política y pública en igualdad de condiciones con las demás, directamente o a través de representantes libremente elegidos, incluidos el derecho y la posibilidad de las personas con discapacidad a votar y ser elegidas".

\section{Aportes e investigaciones desde el campo de la psiquiatría}

La competencia para votar puede ser estudiada como un capítulo dentro de la teoría más amplia de la capacidad para toma de decisiones. Uno de los desarrollos psico-psiquiátricos pioneros en torno a la capacidad para toma de decisiones fue efectuado en 1973 por McGarry-psiquiatra vinculado a la Universidad de Harvard- para instrumentar valoración de la capacidad para estar en juicio. En 1977 Roth, Meisel y Lidz, un grupo de la Universidad de Pittsburgh, publicará el que puede ser considerado el primer test de competencia para consentir tratamiento. En 1982 Appelbaum -en aquel entonces en el Western Psychiatric Institute and Clinic de Pittsburgh- se uniría a Roth para publicar un artículo acerca de la competencia para consentir participar en investigación, en este paper ya comenzarían a quedar sentados los cuatro criterios que habrían de ser los más perdurables y aceptados en cuanto a la capacidad para toma de decisiones: 1-la expresión de una decisión o elección, 2- la comprensión, 3-el manejo racional de la información, y 4- la apreciación de la situación en la que se encuentra o contextual, y se desecharía el criterio relativo a lo 'razonable' de la decisión pensado en tanto su resultado correcto o en la conjetura de lo que haría en su lugar una persona tenida por 'razonable'. Por otro lado, Grisso -psicólogo vinculado a la Universidad de Harvard- trabajó en la elaboración en 1986 de un constructo de 5 componentes: habilidades funcionales, causas (aquí se incluye el diagnóstico, su carácter transitorio o persistente y su impacto sobre las habilidades funcionales), interacción (relativo a la interacción con el contexto), enjuiciamiento y disposición (estas últimas dos resorte de la actividad jurisdiccional del juez) (20, 28-30)

Esas dos líneas de trabajo confluirían y Appelbaum y Grisso desarrollarían los instrumentos estructurados de evaluación de la competencia para toma de decisiones más aceptados y extendidos el MacCAT-T ${ }^{(31)}$ y MacCAT-CR ${ }^{(32)}$, el primero para consentir tratamiento y el segundo para consentir participación en investigaciones. También se desarrolló la MacCAT-Criminal Adjudication para asesorar competencia para estar en juicio en el ámbito penal ${ }^{(33)}$. Una extensa revisión de artículos que se basaron en estas herramientas para sus investigaciones con personas afectadas por esquizofrenia y sujetos no psiquiátricos de control demostró la existencia de una sustancial heterogeneidad en las competencias para toma de decisiones en ambos grupos, hallando que en el primer grupo se determinó un déficit de 
las mismas en el $10-52 \%$ de los sujetos mientras que en el segundo grupo lo hizo en el 0-18\%; por lo tanto, concluyó que el diagnóstico de esquizofrenia no bastaba para presumir incompetencia y a la vez que no todos los sujetos no psiquiátricos poseen habilidades para toma de decisiones intactas ${ }^{(34)}$.

Puede considerarse como iniciático el estudio realizado en 1967 en el Bronx State Hospital de New York que se interesó por la comparación del patrón de voto de los pacientes internados (estudiado a través de unos comicios simulados) y la comunidad del distrito hallando que no había diferencias significativas ${ }^{(35)}$. La misma modalidad se repitió y se publicaron estudios en 1970 en Maryland, en 1977 en Queens-NY, en 1997 en BetYam-Israel y en 2007 en Netanya-Israel arrojando también resultados semejantes entre la población ingresada en hospitales psiquiátricos y la población general ${ }^{(36-39)}$. En un estudio de 2004 sobre pacientes externados viviendo en residencias asistidas en Manheim-Alemania la comparación reflejó una mayor tendencia en el grupo de pacientes a preferir a los denominados left-wingparties, lo que fue interpretado por el autor como consecuencia de sus intereses específicos y de una situación de insatisfacción con su salud ${ }^{(40)}$; y en el mismo sentido de una lectura más detallada de los resultados de los dos estudios producidos en New York en 1967 y $1977^{(35,36)}$ podría desprenderse una leve inclinación a ser más 'democrático-liberales' y menos 'republicano-conservadores'. Entre nosotros, experiencias de comicios simbólicos fueron fue llevadas a cabo por la Asociación Civil La Colifata en el Hospital JT Borda en ocasiones de los comicios entre 1995 y 2007 y nuevamente no se registraron diferencias sustantivas en los resultados según las crónicas periodísticas ${ }^{(41-43)}$.

El interés por las cuestiones vinculadas por la competencia para votar de personas afectadas por trastornos mentales graves, deterioros cognitivos o retrasos madurativos ha ido incrementándose repitiéndose interrogantes sobre la legitimidad o ilegitimidad de su exclusión, el impacto positivo (sobre estos mismos sujetos y sobre la población general en términos de empoderamiento, autoestima, desestigmatización y pluralismo) de la promoción de su inclusión en los procesos electorales, cuál sería el umbral exigible, los problemas de su definición y las oportunidades no discriminatorias para su investigación ${ }^{(44-55)}$. De este devenir ha emergido fortalecida una corriente de opinión que sostiene que el énfasis, más que en la preocupación porque la integridad de las elecciones sea mermada por la participación de estos colectivos, debería comprometerse en extender la participación a los que son competentes para votar y en el riesgo de estar privando del ejercicio de este derecho injusta e ilegítimamente a un grupo de ellos por hacerlo de modo categórico sin un basamento en su habilidad o capacidad focalizada ${ }^{\dagger}$. Una fundación madrileña que ejerce tutela de personas con trastorno mental realizó un estudio mediante las escalas de GENCALT acerca del impacto de su actuación sobre la calidad de vida de 86 de sus tutelados y concluyó que la mejoraba apreciablemente ${ }^{g}$, excepto en los apartados de desarrollo personal y autodeterminación, y que lo hacía con menor eficacia respecto a derechos vinculando esto último a la frecuencia con que las sentencias de incapacitación continuaban privando del derecho al sufragio ${ }^{(56)}$.

Así, en 2005, Appelbaum, Bonnie y Karlawish operacionalizaron una herramienta de entrevista estructurada para evaluación de la competencia para votar (CAT-V) estudiando una población ambulatoria con demencia Alzheimer. Lo realizaron sobre los basamentos teóricos que dieron pie a la serie de MacCAT, utilizando también un sistema de puntuación sin punto de corte y asesorando la performace en las cuatro habilidades básicas para la toma de decisiones: comprensión, apreciación, razonamiento y elección. Sin embargo, formularon dos apartados, un CAT-V "completo" y otro para cumplir los requerimientos del Doe Standard que incluye exclusivamente comprensión de la naturaleza del acto de votar, compresión del efecto del acto de votar y expresión de una elección ${ }^{(57)}$.El denominado Doe Standard es un criterio jurisprudencial establecido por un fallo de la corte federal de Maine en 2001 que dictaminó que no podían considerarse incompetentes para votar a las personas por el mero hecho de hallarse "under guardianship" (figura semejante a la curatela de nuestro medio) por razones de enfermedad mental sino que solo podía hacerlo mediante una evaluación individualizada que determinara "lack the capacity to understand thenature and effect of voting such that they cannot make and individual choice". Estos criterios coinciden con los formulados en los estatutos del Estado de Washington ${ }^{(51)}$. Un grupo de España y un grupo de Italia utilizaron nuevamente versiones del CAT-V para estudiar poblaciones con demencia ${ }^{(58,59,52)}$.

En personas con trastorno mental severo se han conducido dos estudios utilizando la herramienta del CAT-V. El primero de ellos, en 2007, sobre un grupo de 52 pacientes en tratamiento ambulatorio o de Hospital de Día en New York. El 72\% de la muestra tenía un diagnóstico de trastorno psicótico (46\% esquizofrenia) y $20 \%$ de trastorno afectivo mayor. El $90 \%$ de la muestra alcanzó puntuación máxima en el Doe Standard-CAT-V (y $92 \%$ puntuó al menos 5/6). No se halló correlación significativa entre los puntajes en el CAT-V, el deseo de votar o los puntajes en BPRS ${ }^{(60)}$. En el segundo, de 2014 , se estudió una muestra de 56 pacientes internados en un Centro de Salud Mental de Netanya-Israel (abarcando una unidad de larga hospitalización, una guardia cerrada y una guardia gerontopsiquiátrica) y 12 controles sanos. El $78 \%$ de los pacientes reunían criterios diagnósticos de esquizofrenia (y $22 \%$ de 
trastornos afectivos). A través de un análisis de cluster se determinó que el 59\% de los pacientes tenían alta competencia para votar, lo mismo que los 12 controles sanos. Se estableció una correlación inversa significativa respecto a la severidad de la enfermedad (medida con BPRS) y una diferencia significativa entre aquellos pacientes que se encontraban con "guardian" (la figura semejante a la curatela en aquel medio) y aquellos que no ${ }^{(61)}$.

A continuación se ofrece un breve estudio descriptivo retrospectivo con la finalidad de generar datos concretos respecto a las modalidades y resultados que pueden estar implementándose y obteniéndose sobre la materia en nuestro medio.

Comunicación de resultados obtenidos en una muestra de personas afectadas por trastornos psicóticos e incluidas en procesos de determinación de la capacidad jurídica en la provincia de buenos aires

\section{Método}

La muestra de 66 casos para esta comunicación se obtuvo incluyendo a todos los casos con diagnósticos del grupo de los trastornos psicóticos (aquellos que integran el capítulo de Trastornos Psicóticos del CIE-10 y su análogo del DSM-IV) que fueron peritados en el marco de procesos judiciales de Determinación de la Capacidad Jurídica en un Juzgado de Familia de la Departamental Morón de la Provincia de Buenos Aires en el período comprendido desde 01 de marzo de 2014 al 30 de diciembre de 2016. Las evaluaciones fueron realizadas por juntas conformadas por tres médicos psiquiatras o equipo conformado por psiquiatra y asistente social en forma multidisciplinaria o interdisciplinaria, y respecto a la competencia para votar se determinó a través del juicio clínico (aunque no se utilizó la herramienta CATV-Doe Standard se atendió como referencia conceptual a sus criterios).

Tabla 1: Características Sociodemográficas.

\begin{tabular}{|c|c|c|c|}
\hline & & $\mathbf{N}$ & $\%$ \\
\hline \multicolumn{4}{|l|}{ SEXO } \\
\hline & Masculino & 40 & 60,6 \\
\hline 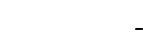 & Femenino & 26 & 39,4 \\
\hline \multicolumn{4}{|l|}{ EDAD } \\
\hline- & Menor a 40 años & 18 & 27,3 \\
\hline- & 40-65 años & 43 & 65,2 \\
\hline & Mayor a 65 años & 5 & 7,5 \\
\hline \multicolumn{4}{|c|}{ INSTRUCCIÓN ALCANZADA } \\
\hline . & Primaria Incompleta & 4 & 6,1 \\
\hline- & Primaria Completa & 35 & 53,0 \\
\hline & Secundaria Completa & 24 & 36,4 \\
\hline- & Universitaria Completa & 3 & 4,5 \\
\hline \multicolumn{4}{|c|}{ Instancia Procesal } \\
\hline & Evaluación & 29 & 43,9 \\
\hline - & Reevaluación & 37 & 56,1 \\
\hline
\end{tabular}

\section{Características sociodemográficas}

La población femenina estuvo subrepresentada. La edad promedio fue de 48,7 años con extremos en 24 y 79 . El 93,9\% de los individuos cumplen criterios de Trastorno Mental Grave según el NIMH (National Mental Healthlnstitute de EEUU). El 92,4\% se encontraba realizando tratamiento psiquiátrico (y el 96,9\% lo había recibido), el $89,4 \%$ cursó al menos una internación psiquiátrica y el 22,7\% se encontraba alojado en una clínica psiquiátrica o un hogar geriátrico. Solo cuatro participantes $(6,1 \%)$ realizaban al momento de su evaluación tareas remuneradas y competitivas. El 74,2\% de la muestra posee un diagnóstico de esquizofrenia y un $18,2 \%$ de trastorno psicótico no especificado. 
Tabla 2: Características Psiquiátricas.

\begin{tabular}{|c|c|c|c|}
\hline & & $\mathbf{N}$ & $\%$ \\
\hline \multicolumn{4}{|c|}{ DIAGNÓSTICO } \\
\hline- & ESQUIZOFRENIA & 49 & 74,2 \\
\hline- & _Esq. Paranoide & 21 & 31,8 \\
\hline- & _Esq. Hebefrénica & 19 & 28,8 \\
\hline- & _Esq. Residual & 7 & 10,6 \\
\hline- & _Esq. Indiferenciada & 2 & 3,0 \\
\hline- & T. PSICÓTICO NO ESPECIFICADO & 12 & 18,2 \\
\hline- & T. IDEAS DELIRANTES & 3 & 4,5 \\
\hline- & T. ESQUIZOAFECTIVO & 2 & 3,0 \\
\hline \multicolumn{4}{|c|}{ TRATAMIENTO PSIQUIÁTRICO } \\
\hline- & Actual & 61 & 92,5 \\
\hline- & En el pasado & 3 & 4,5 \\
\hline- & Nunca & 2 & 3,0 \\
\hline \multicolumn{4}{|c|}{ Cursó al menos una Internación Psiquiátrica } \\
\hline- & Sí & 59 & 89,4 \\
\hline- & NO & 7 & 10,6 \\
\hline \multicolumn{4}{|c|}{ Ingresados en Clínica Psiquiátrica u Hogar Geriátrico a la fecha del examen } \\
\hline- & Sí & 15 & 22,7 \\
\hline- & No & 51 & 77,3 \\
\hline
\end{tabular}

Tabla 3: Competencia para Votar.

\begin{tabular}{|c|c|c|c|c|c|}
\hline \multicolumn{2}{|l|}{} & Sí $(\mathbf{N})$ & SÍ $(\%)$ & NO $(\mathbf{N})$ & NO $(\%)$ \\
\hline COMPETENCIA PARA VOTAR & 33 & 50,0 & 33 & 50,0 \\
\hline Según Diagnóstico & 22 & 44,9 & 27 & 55,1 \\
\hline - ESQUIZOFRENIA & 9 & 42,9 & 12 & 57,1 \\
$-\quad$ Esq. Paranoide & 10 & 52,6 & 9 & 47,4 \\
$-\quad$ Esq. Hebefrénica & 1 & 14,3 & 6 & 85,7 \\
$-\quad$ Esq. Residual & 2 & 100 & 0 & 0 \\
$-\quad$ Esq. Indiferenciada & 7 & 58,3 & 5 & 41,7 \\
$-\quad$ T. PSICÓTICO NO ESPECIFICADO & 2 & 66,7 & 1 & 33,3 \\
$-\quad$ T. ESQUIZOAF DELIRANTES & 2 & 100 & 0 & 0 \\
\hline Ingresados en Clínica Psiquiátrica u Hogar Geriátrico & 3 & 20,0 & 12 & 80 \\
\hline No Ingresados en Clínica Psiquiátrica u Hogar Geriátrico & 30 & 58,8 & 21 & 41,2 \\
\hline
\end{tabular}

\section{Resultados}

Se determinó que el $50,0 \%$ de la muestra exhibía competencia para votar. Dicho porcentaje se incrementó al $58,8 \%$ si se recorta la muestra a aquellos individuos que no se encuentran ingresados en instituciones (los 12 individuos alojados en Clínicas Psiquiátricas u Hogares Geriátricos exhibieron una tasa de no competencia para votar sustancialmente más elevada -80\%-). Se observó heterogeneidad en los resultados de competencia para votar obtenidos para los diferentes grupos diagnósticos: 44,9\% en esquizofrenia -a su vez, 52,6\% en la forma clínica hebefrénica, 42,9\% en la forma clínica paranoide y $14,3 \%$ en la residual-, $58,3 \%$ en trastorno psicótico no especificado, $66,7 \%$ en trastorno de ideas delirantes y $100 \%$ en trastorno esquizoafectivo (en estos últimos dos grupos con una muestra demasiado exigua de sólo 3 y 2 individuos respectivamente). De los 37 procedimientos en los que se trató de una 
reevaluación (ordenada ya fuera por artículo 152 ter del Código Civil modificado por ley 26.657 o por artículo 40 del nuevo $\mathrm{CCyCN}$ ), la evaluación previa había arrojado sus resultados bajo la rúbrica de demente jurídico y/o compatible con el artículo 141 de nuestro antiguo Código Civil en casi la totalidad, $36(97,3 \%)$, de ellos -el caso restante había sido hallado compatible con el artículo 152bis-; y de estos 18 , el 50,0\%, fueron encontrados competentes para votar en la reevaluación ingresada a nuestro estudio.

\section{Discusión}

Las principales limitaciones de este estudio provienen del posible sesgo de los evaluadores y la limitada representatividad de la muestra. Por lo tanto, no puede obviarse el reconocimiento de dicha carencia aunque esta pueda ser parcialmente contenida por la participación en juntas de tres psiquiatras conformadas por el autor junto a otros cinco colegas de amplia experiencia en el fuero de Familia o junto a trabajadores sociales de las mismas condiciones actuando consensuada y deliberativamente. Coincidimos con Calcedo Barba ${ }^{(20)}$ en que "las habilidades del votante solo pueden ser consideradas de modo formal, nunca sustantivo" 250p. Y encontramos que la evaluación de las competencias para la toma de decisiones de una persona debe ser prudencial, razonada, probabilística y deliberativa, no de certeza científica, y si bien las herramientas o protocolos desarrollados pueden ser de utilidad, al usarlos, aun siendo validados, habrá que asumir la posibilidad de equivocarse técnica y éticamente. Instrumentos como el CAT-V reconocen explícitamente las limitaciones derivadas de la no existencia de umbrales o puntos de corte y el carácter orientativo de sus puntuaciones y el patrón oro de comparación continua siendo el juicio de expertos experimentados ${ }^{(28-30,62)}$. Al mismo tiempo, es menester señalar que en nuestro medio no existe una definición jurisprudencial como la establecida en el Doe Standard, y que podrá optarse por dicha referencia como guía ante la incipiente aceptación internacional que se le tributa a la herramienta que lo operacionaliza (en virtud del uso en estudios efectuados en España, EEUU, Israel e Italia del CAT-V-Doe Standard y el asentado reconocimiento a los modelos de los que deriva) y el acuerdo axiológico con su permisividad para no privar innecesariamente a individuos de sus derechos y su suficiencia restrictiva para proteger la integridad del voto.

En un estudio de la población con trastornos psicóticos en procesos de incapacitación civil de un Juzgado de Internamientos e Incapacidades de Madrid durante el período junio 95-mayo 96 ( $n=30$ ) se arribó coincidentemente a un porcentaje de diagnóstico de esquizofrenia en el $73,3 \%$ de los casos; sin embargo, no se formularon diagnósticos de trastorno psicótico no especificado y el $26,7 \%$ restante se repartió entre trastorno por ideas delirantes persistentes $(20,0 \%)$ y trastorno esquizoafectivo $(6,7 \%)$. No se informó específicamente sobre competencia para votar, pero se señaló que en el $50 \%$ de los casos se determinaba incapacidad parcial, en el $40 \%$ incapacidad total y en el $10 \%$ capacidad $^{(63)}$.

Al igual que en otros estudios ${ }^{(60,61)}$ encontramos que un porcentaje muy importante de personas afectadas por trastornos psicóticos, incluso en una muestra como la nuestra ceñida a personas incluidas en procesos judiciales de determinación de la capacidad jurídica, son valoradas como competentes para votar. Y observamos que su determinación como una competencia específica dentro de reevaluaciones de personas con trastornos mentales graves que se encontraban bajo curatela del antiguo artículo 141 permite detectar que un número relevante de ellas también resultarían competentes para ejercer su derecho al sufragio activo.

\section{Conclusiones}

La definición de criterios de competencia para votar (y de la oportunidad o no de instrumentar medidas para su evaluación) posee un carácter netamente político que excede las posibilidades de la ciencia.

Desde la psiquiatría pueden aportarse elementos que incidan cuestionando la legitimidad de la exclusión del ejercicio del derecho al sufragio a partir del diagnóstico de un trastorno mental grave, de una situación de internación o de un estatus genérico de incapacitación civil.

\section{Bibliografía}

1. Toro Martínez E. Capacidad y riesgo en la Ley de Salud Mental y su Decreto de Reglamentación. Luces y escotomas del nuevo paradigma. Psiquiatría Forense, Sexología y Praxis. 2013; 8:188-200; p. 193.

2. Achaval A. Psiquiatría médicolegal y forense. Buenos Aires: Ed. Astrea; 2003.

3. Zazzali JR. La pericia psiquiátrica. Buenos Aires: Ed. La Rocca, 2da. Ed.; 2013.

4. Cástex MN. Insania e Inhabilitación. Buenos Aires: Ed. Ad-Hoc; 2009.

5. Betta JC. Psicopatología Forense. Buenos Aires: Centro Editor Argentino; 2da. Ed, 2003.

6. Dirección Nacional de Salud Mental y Adicciones. Instrumento de Relevamiento - Capacidad Jurídica. Buenos Aires; 2012.

7. Organización Mundial de la Salud. Clasificación Internacional del Funcionamiento, de la Discapacidad y de la Salud de la Organización Mundial de la Salud (CIF-OMS). Madrid: OMS; 2001. 
8. De Veyga F. Estudios Médico Legales sobre el Código Civil Argentino. Buenos Aires: Ed. Librería Científica de Agustín Etchepareborda; 1900.

9. Rojas N. Medicina Legal. Buenos Aires: Ed. El Ateneo, 3ra. Ed; 1947.

10. Bonnet EFP. Medicina Legal. Buenos Aires: Ed. López Libreros, 2da. Ed.; 1980.

11. Raitzin A. Las pericias médico-legales sobre alienados. Buenos Aires; 1929.

12. Lucero AL. Psicopatología Forense, informes en materias criminal y civil. Buenos Aires: Ed. Universidad de Tucumán; 1917.

13. Ruiz Maya M. Psiquiatría Penal y Civil. Madrid: Ed. Plus-Ultra; 1931.

14. Weygandt W. Psiquiatría Forense. Barcelona: Ed. Labor; 1928.

15. De Sanctis S, Ottolenghi S. Trattato Pratico di Psicopatologia Forense per uso deimedici, giuristi e student. Milano: Società EditriceLibraria; 1920; pp. 1179-1181.

16. Claude H. Psiquiatría Médico-Legal. Madrid: Ed. Espasa-Calpe; 1933; p.8.

17. De Veyga F. Degeneración y degenerados. Miseria, vicio y delito. Buenos Aires: Ed. El Ateneo, Buenos Aires; 1938.

18. Llambías JJ. Tratado de Derecho Civil. Buenos Aires: Ed. Abeledo Perrot; 2003.

19. Rojas N. La Psiquiatría en la Legislación Civil. Buenos Aires: Ed. Valerio Abeledo; 1938.

20. Calcedo Ordoñez A. La pericia médica en el procedimiento de incapacitación civil. En: Los derechos de las personas con discapacidad. Madrid: Ed. Consejo Superior del Poder Judicial; 2007.

21. Esbec Rodríguez E. Avances y Exigencias de la Prueba Pericial en el Proceso de Modificación y Delimitación de la Capacidad de Obrar. La Capacidad en los Trastornos Mentales. Psicopatología Clínica Legal y Forense. 2012; 12:149179.

22. Palacios A. El modelo social de discapacidad: orígenes, caracterización y plasmación en la Convención Internacional sobre los Derechos de las Personas con Discapacidad. Madrid: Grupo Editorial CINCA; 2008.

23. Martínez MJ, Daray FM. Interdicción e inhabilitación: consideraciones acerca de su evolución normativa y conceptual. Alcmeon Revista Argentina de Clínica Neuropsiquiátrica. 2012; 17:249-259.

24. Carrillo R. Clasificación Sanitaria de los Enfermos Mentales. Relaciones entre Código Civil y Sanitario. En: Obras Completas, t. V. Buenos Aires: Ed. EUDEBA; 1974.

25. Comité sobre los Derechos de las Personas con Discapacidad de las Naciones Unidas. Observaciones finales sobre el informe inicial de Argentina. 2012. Disponible el 02/08/2016 en: www.ohchr.org/Documents/HRBodies/CRPD/8thSession/CRPD-C-ARG-CO-1_sp.doc

26. CIV 83563/1997/CS1 "F , H O si artículo 152 ter. Código Civil"; 2016, accessible el 02/08/2016 en http://www.fiscales.gob.ar/wp-content/uploads/2016/04/CIV_83563_1997_F.pdf

27. Beckman L. The Accuracy of Electoral Regulations: The Case of the Right to Vote by People with Cognitive Impairments. Social Policy and Society. 2014; 13: 221-233.

28. Simón Lorda P. La capacidad de los pacientes para tomar decisiones: una tarea todavía pendiente. Revista de la Asociación Española de Neuropsiquiatría. 2008; 28:325-348.

29. Navio Acosta M, Ventura Faci T. Manual de Consulta en Valoración de la Capacidad. Madrid: Editorial Médica Panamericana; 2014.

30. Ventura Faci T, Navio Acosta M, Álvarez Marrodán I, Baón Pérez B. La evaluación de la capacidad y sus problemas. EIDON. 2014; 41:12-27.

31. Grisso T, Appelbaum PS. The MacArthur Competence Assessment Tool - Treatment (MacCAT-T). Sarasota (FL USA): Professional Resources Press; 1998.

32. Appelbaum PS, Grisso T. The MacArthur Competence Assessment Tool - Clinical Resarch (MacCAT-CR). Sarasota (FL - USA): Professional Resources Press; 2000.

33. Hoge SK, Bonnie RJ, Poythress N, et al. The MacArthur Competence Assessment Tool-Criminal Adjudication. Odessa (FL - USA): Psychological Assessment Resources; 1999.

34. Jeste DV, Depp CA, Palmer BW. Magnitude of Impairment in Decisional Capacity in People With Schizophrenia Compared to Normal Subjects: AN Overview. Schizophrenia Bulletin. 2006; 32:121-128.

35. Klein M, Grossman SA. Voting Pattern of Mental Patients in a community State Hospital. Community Mental Health Journal. 1967; 3:149-152.

36. Wellner AM, Gaines LS. Patients Right to Vote. Hospital \& Community Psychiatry. 1970; 21:163-164.

37. Howard G, Anthony $R$. The right to vote and voting patterns of hospitalized psychiatric patients. Psychiatric Quarterly. 1997; 49:124-32.

38. Melamed Y, Shamir E, Solomon Z, Elizur A. Hospitalized mentally ill patients vote for the first time. Israel Journal of Psychiatry and Related Sciences. 1997; 34:69:72.

39. Melamed Y, Doron A, Finkel B et al. Israeli Psychiatric Inpatients Go to the Polls. Journal of Nervous \& Mental Disease. 2007; 195:705-708

40. Bullenkamp J, Voges B. Voting Preferences of Outpatients With Chronic Mental IIInes in Germany. Psychiatric Services. 2004; 55: 1440-1442.

41. Coincidencia. Página 12. 15 de Mayo de 1995.

42. La Colifata TV 2003 video disponible enhttps://www.youtube.com/watch?v=-Nxc3jDLiEA el 02/08/16

43. La cábala del Borda. Página 12. 28 de Octubre de 2007.

44. Jaychuk G, Manchanda R. Psychiatric patients and the federal election. Canadian Journal of Psychiatry. 1991; 36:124125.

45. Humphreys M. Getting psychiatric patients to the polls in the 1992 General Elections. Psychiatric Bulletin. 1993; 17, 1819.

46. Melamed Y, Solomon Z, Elizur A. Voting by Israeli Patients. Psychiatric Services. 1997; $48: 1081$.

47. Appelbaum PS. "I Vote. I count": Mental Disability and the Right to Vote. Psychiatric Services. 2000; 51:849-850, 863. 
48. Melamed Y. Hospitalised mentally ill patients vote in Israel. Journal of Medical Ethics. 2001; 25:355.

49. Karlawish JH, Bonnie RJ, Appelbaum PS, et al. Addressing the ethical, legal, and social issues raised by voting by personas with dementia. JAMA. 2004; 292:1345-1350.

50. Irastorza LJ Competencia para votar y trastorno mental. Internet. 7mo. Congreso Virtual de Psiquiatría Interpsiquis; Febrero de 2006.

51. Hurme SB, Appelbaum PS. Defining and assessin capacity to vote: the effect of mental impairment on the right of voters. McGeorge Law Reviews. 2007; 38:931-979.

52. Tiraboschi P, Chitò E, Sacco L, et al. Evaluating Voting Competence in Persons with Alzheimer Disease. International Journal of Alzheimer's Disease; 2011. Disponible el 02/08/2016 en: http://www.ncbi.nlm.nih.gov/pmc/articles/PMC3139143/pdf/IJAD2011-983895.pdf

53. McIntyre J, Khwaja M, Yelamanchili V, et al. Uptake and knowledge of voting rights by adult in-patients during the 2010 UK general election. ThePsychiatrist. 2012; 36:126-130.

54. Melamed Y, Donsky L, Oyffe I et al. Voting of Hospitalized and Ambulatory Patients with Mental Disorders in Parliamentary Elections. Israel Journal of Psychiatry and Related Sciences. 2013; 50: 13-16.

55. Feinstein M, Webber D. Voting under guardianship: Individual rights requires individual review. NAELA Journal. 2014; 10:125-148.

56. Pallarés J, Perdigón AL, Molinero MC et al. Evaluación de la calidad de vida en una muestra de personas con enfermedad mental incapacitadas judicialmente. Psicopatología Clínica, Legal y Forense. 2014; 14:79-95.

57. Appelbaum PS, Bonnie RJ, Karlawish JH. The Capacity to Vote of Personas With Alzheimer's Disease. American Journal of Psychiatry. 2005; 162:2094-2100.

58. Irastorza LJ, Corujo P, Bañuelos P. Capacity to Vote in Persons with Dementia and the Elderly. International Journal of Alzheimer's Disease; 2011. Disponible el 02/08/2016 en: https://www.hindawi.com/journals/ijad/2011/941041/

59. Irastorza LJ, Corujo P, Bañuelos P. Competencia para votar en pacientes con demencia. Revista de Neurología. 2007; 44: $321-5$.

60. Raad R, Karlawish J, Appelbaum PS. The Capacity to Vote of Persons With Serious Mental IIIness. Psychiatric Services. 2009; 60:624-628.

61. Doron A, Kurs R, Stolovy T, et al. Voting Rights for Psychiatric Patients: Compromise of the Integrity of Elections, or Empowerment and Integration into the Community? Israel Journal Psychiatry Related Science. 2014; 51:169-174.

62. Villagran JM, Lara Ruiz-Granados I, González-Saiz F. El proceso de decisión compartida en el tratamiento del paciente psiquiátrico: estudios empíricos y evaluación de capacidad. Revista de la Asociación Española de Neuropsiquiatría. 2014; 34:491-506.

63. Delgado Bueno S. Evaluación de la capacidad de obrar. Psicopatología Clínica, Legal y Forense. 2004; 4:97-133.

a Manuel Ruiz Maya: Notable psiquiatra español sobre quien hizo reiteradas menciones elogiosas Nerio Rojas. Su carrera fue trágicamente truncada al ser fusilado por los sublevados franquistas en agosto de 1936 en la Córdoba andaluza.

b El libro I del CC donde se ubica el artículo 141 había sido completado ya en 1965 y la filiación de este artículo es propiamente legal, no psiquiátrica, como deja traslucir su inexacto uso de la terminología médica (en su nota sobre el art. 3615 el autor aclara su uso: 'demencia es la expresión genérica que designa a todas las variedades de la locura'), y Vélez Sársfield abrevó en las fuentes del Code Civil des français y el Esboço do Codigo Civil de Teixeira Freitas. Pese a ello, rastrear y repasar sus textos ("Art. 489 Le majeur quiest dans un état habituel d'imbecilité, de démence ou de fureur, doit être interdit, même lorsque cet état presente des intervalles lucides." y "Art. 79. Declarar-se-á como alienados os individuos de un e outro sexo, que se acharem em estado habitual de mania, demencia ou imbecilidade; ainda mesmo que tenham lúcidos intervalos, ou mania pareça parcial."), no deja de tener un interés para la historia y epistemología psiquiatría al revelar en la plasmación de textos jurídicos el movimiento sucesivo en el que el potencial reconocimiento de una manía parcial pasa de inicialmente no ser considerado, luego a ser considerado como una apariencia (pareça), y finalmente ser considerado con plenitud pero a la vez que se soslaya que pudiera generar consecuencias diferenciadas. Éste debe ser situado en la 2da. mitad del siglo XIX en la transición desde el alienismo inicial al paradigma de las enfermedades mentales, uno de cuyos hitos -a la par de la introducción de la diacronía- operados por JP Falret es el rechazo de la posibilidad de coexistencia de la locura y la cordura. El género monomanía creado por J Esquirol resumía las "locuras parciales" o "los delirios parciales" y su discípulo Falret lo atacaría frontalmente con su Sobre la no existencia de la monomanía de 1854.

c Si bien es aún asunto de debate por su frecuente utilización indistinta con capacidad y su posible traducción cruzada con la literatura norteamericana, entendemos con Toro Martínez y Esbec Rodríguez que el término más adecuado para el ámbito médico-legal es el de competencia (por ejemplo competencia para votar, competencia para otorgar testamento o competencia para consentir participar en investigaciones médicas) y que a la vez éste tiene potencial para promover el enfoque individualizado de funcionalidad y especificidad.

d A partir de entonces se acciones encaradas por actores como el Ministerio Público de la Defensa, el INADI, La Colifata, AC Cooperanza y ACIJ promovieron y facilitaron la participación en los comicios de numerosas personas internadas de larga data en los hospitales BA Moyano y JT Borda entre otros hospitales monovalentes.

e La lista completa: Austria, Canadá, Bolivia, Croacia, Ecuador, Eslovenia, Finlandia, Holanda, Irlanda, Israel, Italia, Kenia, Méjico, Noruega, Reino Unido y Suecia.

f Para calibrar las características de esta corriente tal vez sea de utilidad resaltar que, excediendo controversias locales y más allá de actores jurídicos, de la ciencia política y de la sociedad civil, la temática ha formado parte insistentemente de la agenda de trabajo de referentes como PS Appelbaum quien por fuera de posiciones radicales o antipsiquiátricas ha ejercido la presidencia de la American Psychiatry Asociation durante 2002-2003.

g Incluida la dimensión 'Bienestar físico' que abarca los indicadores de Atención Sanitaria, Sueño, Salud y sus alteraciones, Actividades de la vida diaria, Acceso a ayudas técnicas y Alimentación. 